\title{
Interface of Female Sexual Dysfunction, Women's Mental Health, and Psychiatry
}

\author{
Darpan Kaur
}

\begin{abstract}
Literature highlights that globally, female sexual dysfunction (FSD) is highly prevalent and has relevant interface with psychiatric disorders and women's mental health. It is described to have complex biopsychosocial etiopathogenesis. Psychiatric disorders such as mood disorders, schizophrenia, substance use disorders, anxiety disorders, eating disorders, and personality disorders in women are described to have high comorbidity with FSD. Certain medical conditions have also been linked to FSD. Neurobiological and genetic studies have highlighted novel mechanisms for FSD. The clinical assessment of FSD needs detailed evaluation and special diagnostic and interview techniques. Management of FSD poses unique challenges with currently limited evidence base for psychopharmacological and psychotherapy management.

Keywords: Female sexual dysfunction, Psychiatric disorders, Women's mental health.

Indian Journal of Private Psychiatry (2019): 10.5005/jp-journals-10067-0028
\end{abstract}

\section{INTRODUCTION}

Globally, female sexual dysfunction (FSD) has emerged as an important problem that needs to be addressed for enhanced clinical care. ${ }^{1}$ Decreased desire for sexuality has been reported as the most common complaint among women experiencing sexual dysfunction. ${ }^{2}$ Studies have found that up to $43 \%$ of women can be affected with FSD. ${ }^{3}$ Some studies highlight that the prevalence can vary from $20 \%$ to $50 \%$ with various factors such as data collection instruments, cutoffs use, cultural factors, diverse definitions, etc., affecting the overall reported prevalence in literature. ${ }^{4}$

\section{Definition}

Female sexual dysfunction can be defined as a disorder of sexual desire, arousal, or orgasm, and/or sexual pain, which can lead to personal distress as well as impact the quality of life and personal relationships. ${ }^{5}$

\section{Classification}

There is no separate category of FSD in the ICD 10, and it describes sexual dysfunction in the broader F52 category with heading of sexual dysfunction not caused by organic condition. There is description of lack or loss of desire, sexual aversion and lack of sexual enjoyment, failure of genital response, orgasmic dysfunction, nonorganic vaginismus and nonorganic dyspareunia, and excessive sexual desire. ${ }^{6}$ DSM-5 mentions female sexual interest/ arousal disorder, female orgasmic disorder, and genitopelvic pain/ penetration disorder as subtypes of FSD. ${ }^{7}$

\section{Neurobiology and Genetics}

Female sexual dysfunction is considered to be very complex etiopathologically and no single cause can be pinpoint attributed to the causation with absolute certainty. ${ }^{8}$ Neurobiological studies describe that hypoactive sexual desire disorder can have an association with fronto-limbic-parietal dysfunction with problems in neurocircuitry of sexual desire especially at the brain neuronal network and the self-referential brain network. ${ }^{9}$ Neurogenetics studies in FSD highlight that THNSL2 and SLC6A19, which have been linked to weight and adiposity, might represent novel candidates
Department of Psychiatry, Mahatma Gandhi Mission Medical College, Navi Mumbai, Maharashtra, India

Corresponding Author: Darpan Kaur, Department of Psychiatry, Mahatma Gandhi Mission Medical College, Navi Mumbai, Maharashtra, India, Phone: +91 9769634045, e-mail: kaur.darpan@gmail.com

How to cite this article: Kaur D. Interface of Female Sexual Dysfunction, Women's Mental Health, and Psychiatry. Ind J Priv Psychiatry 2019;13(1):7-10.

Source of support: Nil

Conflict of interest: None

for sexual problems in women and an interesting association of a shared genetic common component between anxiety sensitivity and FSD. ${ }^{10}$ The putative role of the exon 3 repeat region, and the C-521T and C-616G promoter region SNPs and a consistent role for dopamine specifically DRD4 receptor gene expression and protein concentrations at the neuroreceptor-based genetics has been described in literature. ${ }^{11}$

\section{FSD in Youth and Elderly}

There have been studies highlighting the role of hormones, menopause, ageing, metabolic factors, and psychosocial stressors modulating FSD in elderly. ${ }^{12}$ Contrary to the popular belief, FSD is even affecting young women especially with confounding factors of severity of psychopathology, use of certain psychiatric medications, lower medication adherence, and negative perceived experiences related to sexuality ${ }^{13}$ Women above 40 years of age have described more problems with sexuality especially in clinic-based studies. ${ }^{14}$ Sexual functioning-related changes with the ageing process in women were more described to be in the desire and arousal domain in certain literature. ${ }^{15}$

\section{Women's Mental Health, Psychosocial FACTORS, AND FSD}

There has been a role of cultural factors, stigma, psychosocial factors, gender inequality, beliefs, and taboos, which can lead 
to underexpression of sexual problems in women as well as underrecognition and undertreatment. ${ }^{16}$ Stressors in relationship, marriage, sexual victimization, emotional abuse, violence, childhood sexual abuse, and trauma in the past and ongoing trauma have been found to have correlations with FSD. ${ }^{17-19}$

\section{Psychiatric Disorders and FSD Depression and FSD}

Certain studies have found very high prevalence of FSD with depression as high as $90 \%$ regardless of the type and severity of depression. Depression associated with medical comorbidities further enhances problems with sexual functioning in women. ${ }^{20}$

\section{Substance Use and FSD}

Studies have reported high prevalence of $34.2 \%$ in patients who take substances, and severe nicotine dependence has been described to increase the chances of having sexual dysfunction by 2.72 -fold in women. ${ }^{21}$ Literature recognizes that women with mood, anxiety, and substance dependence have elevated scores on problems with sexual satisfaction even after controlling for confounders. Bipolar disorders and comorbid alcohol and drug dependence reduce sexual satisfaction in women. ${ }^{22-25}$

\section{Schizophrenia and FSD}

Studies have highlighted the association of schizophrenia in women and sexual dysfunction in as high as $70 \%$ of patients. It was further found that impaired desire was the most common problem reported by all women. ${ }^{26}$

\section{Bipolar Disorders and FSD}

Sexual problems have also been studied in women with bipolar disorders and they have been associated with mood, cognitions, and perceptions. ${ }^{27}$

\section{Personality and FSD}

The role of personality, negative coping, introversion, not being open to new experiences, and emotional instability has been described in women with FSD. ${ }^{28}$

\section{Obsessive Compulsive Disorder and FSD}

Female patients with obsessive compulsive disorder (OCD) also reported sexual avoidance (60.6\%) and anorgasmia (24.2\%), and literature also describes sexual dysfunction unrelated to pharmacotherapy in patients with OCD. ${ }^{29}$

\section{Anxiety Disorder and FSD}

Sexual dysfunction was found to occur in $64 \%$ of women with panic disorders. ${ }^{29}$ Sexual dysfunction has also been described in women with anxiety disorders related to the cognitive and neurobiological threat of performance anxiety. ${ }^{30}$

\section{Eating Disorders and FSD}

Eating disorders have also been linked to problems with sexual functioning in women. ${ }^{31}$

\section{Drug-related Sexual Dysfunction}

Certain drugs can affect sexual function through multiple pathways. Drugs that affect the central serotonin, dopamine, and/ or prolactin signaling pathways have been putated to play an important role. ${ }^{32}$ Literature highlights that a significant percentage of women taking medications report varied levels of problems in sexual functioning. ${ }^{33}$ Postsynaptic dopamine antagonism, prolactin elevation, and a1-receptor blockade may have a role in antipsychotic-induced sexual dysfunction. ${ }^{34}$ Psychotropic drugs use can cause associated sexual dysfunction and these can affect compliance and adherence. ${ }^{35}$ However, certain literature highlights that female gender along with depression can increase the association of FSD regardless of the type of drug use with putative role of cognitions at the neurobiological level. ${ }^{36-38}$

\section{Multiple FSD}

Literature has consistently highlighted that problems in desire or arousal are most common; however, emerging literature highlights that many women in significant numbers experience multiple sexual dysfunctions. ${ }^{17}$

\section{Medical Conditions and FSD}

Certain medical conditions have been linked to FSD including atherosclerosis, peripheral arterial disease and hypertension, posthysterectomy, heart disease, hypertension, diabetes, obesity, smoking, stroke, polycystic ovary syndrome, and metabolic syndrome. ${ }^{39}$

\section{Clinical Assessment in FSD}

Detailed history taking in psychiatry and clinical interview forms a very vital part in diagnosis and clinical management of patients experiencing sexual problems. It is suggested that the clinical history should assess more about clinical aspects of FSD such as (a) generalized or situational; (b) lifelong or acquired; (c) the level of distress and impact of FSD on personal life; and (d) the leading etiologies. ${ }^{24}$ It is recommended that clinicians explore attachment styles of patients, childhood experiences, abuses, onset of sexual activity, personality, cognitive schemas, infertility concerns, and sexual expectations. Assessment of psychiatric comorbidity should be carried as part of the detailed evaluation. ${ }^{2}$ The role of relationships, marital life, familial, work-related stressors, negative coping, medical comorbidity, existing medications, and substance use history must also be explored. Laboratory assessments for appropriate medical comorbidity known to be associated in FSD based on age and individual medical history can include complete blood count, metabolic workup, screening for diabetes, dyslipidemia, thyroid dysfunction, and nutritional deficiencies, which may be done based on reviewing available literature and the clinical profile of the patient. Certain literature highlights need for endocrinological and gynecological liaison workup in certain cases and need for hormonal and metabolic profile assessment as indicated on an individual case-to-case basis. The International Consultation on Sexual Medicine Group has proposed an updated algorithm for diagnostic evaluation of FSD with specific recommendations for sexual history taking and diagnostic evaluation. It also recommends the use of standardized scales, checklists, and validated questionnaires in routine clinical care. ${ }^{40}$ It was also found that low sexual desire was more specifically associated with levels of free testosterone and androstenedione, but FSD in general was not associated with androgen levels. ${ }^{41}$

\section{Pharmacotherapy}

The hormone therapy, including estrogens, testosterone, tibolone, and dehydroepiandrosterone, has been tried in certain studies 
of postmenopausal women, which have discussed the potential use of on-demand combined hormonal (testosterone) and nonhormonal (buspirone or sildenafil) treatments to address possible neurophysiological profiles of women. ${ }^{42}$ Flibanserin, a centrally acting medication targeting the serotonin, dopamine, and norepinephrine systems, has received FDA approval for low sexual desire in women. ${ }^{2}$ There is lack of randomized clinical trials assessing the effects of switching to currently available antidepressant agents with lower rates of adverse sexual effects, the role of psychological or mechanical interventions, or of techniques such as drug holidays. The addition of bupropion at higher doses has been shown to be useful for women experiencing selective serotonin reuptake inhibitors (SSRI)-related sexual dysfunction. ${ }^{43}$ Sildenafil has been studied in treatment of antipsychotic-induced sexual dysfunction in men with schizophrenia but has not been studied in women with FSD as per higher levels of evidence. Further well-designed randomized control trials addressing these critical psychopharmacological aspects are needed. ${ }^{35}$ Treatment of adverse sexual effects can be pharmacological (dose reduction, drug discontinuation or switching, augmentation, or using medications with lower adverse effect profiles). ${ }^{33}$ There is not adequate evidence to support the use of oxytocin or progesterone for FSD. There is some literature that suggests that treating hyperprolactinemia might be helpful in reducing FSD. ${ }^{39}$

\section{PSYCHOTHERAPY}

Literature highlights that certain psychotherapies such as behavior therapy, Master and Johnson therapy, Jacobson progressive muscle relaxation, cognitive behavior therapy (CBT), psychoeducational therapies, exercise, and stimulation-based techniques have been tried and found to be useful in varied levels of evidence. ${ }^{33}$

\section{IMPLICATIONS}

Biopsychosocial frameworks of treatment with holistic focus on medical, psychiatric, and psychosocial factors need to be considered for enhanced clinical care of patients with FSD. ${ }^{44}$ Literature recommends that medical professionals must be welltrained and sensitive to women suffering from FSD and be aware of diverse intersections with psychiatric disorders in women. ${ }^{45}$

\section{Conclusion}

Female sexual dysfunction is an arena that intersects with psychiatry and women mental health and it still remains high in occurrence yet underrecognized and undertreated. Training and creating additional awareness in the domain of FSD and its intersection with psychiatric disorders is recommended. ${ }^{46}$

\section{References}

1. Wright JJ, O'Connor KM. Female sexual dysfunction. Med Clin North Am 2015;99(3):607-628. DOI: 10.1016/j.mcna.2015.01.011.

2. Brotto LA. Evidence-based treatments for low sexual desire in women. Front Neuroendocrinol 2017;45:11-17. DOI: 10.1016/ j.yfrne.2017.02.001.

3. Crisp CC, Vaccaro CM, Pancholy A, et al. Is female sexual dysfunction related to personality and coping? An exploratory study. Sex Med 2013;1(2):69-75. DOI: 10.1002/sm2.16.

4. Garcia S, Moreno S, Aponte H. Prevalence of sexual dysfunction in female outpatients and personnel at a Colombian hospital: correlation with hormonal profile. J Sex Med 2008;5(5):1208-1213. DOI: 10.1111/j.1743-6109.2007.00718.x.
5. Walton B, Thorton T. Female sexual dysfunction. Curr Womens Health Rep 2003;3(4):319-326.

6. ICD 10 Version 2016. http://apps.who.int/classifications/icd10/ browse/2016/en\#!/F52.0.

7. IsHak WW, Tobia G. DSM-5 changes in diagnostic criteria of sexual dysfunctions. Reprod Sys Sexual Disord 2013;2:122 https://www. omicsonline.org/dsm-5-changes-in-diagnostic-criteria-of-sexualdysfunctions-2161-038X.1000122.php?aid=18508.

8. Burri A, Leupin M, Spector T, et al. Differential DNA methylation in monozygotic twins discordant for female sexual functioning. J Sex Med 2017;14(11):1357-1364. DOI: 10.1016/j.jsxm.2017.09.003.

9. Cacioppo $S$. Neuroimaging of female sexual desire and hypoactive sexual desire disorder. Sex Med Rev 2017;5(4):434-444. DOI: 10.1016/ j.sxmr.2017.07.006.

10. Burri A, Spector T, Rahman Q. The etiological relationship between anxiety sensitivity, sexual distress, and female sexual dysfunction is partly genetically moderated. J Sex Med 2012;9(7):1887-1896. DOI: 10.1111/j.1743-6109.2012.02710.x.

11. Ben Zion IZ, Tessler R, Cohen L, et al. Polymorphisms in the dopamine D4 receptor gene (DRD4) contribute to individual differences in human sexual behavior: desire, arousal and sexual function. Mol Psychiatry 2006;11(8):782-786. DOI: 10.1038/sj.mp.4001832.

12. Nappi RE, Martini E, Martella S, et al. Maintaining sexuality in menopause. Post Reprod Health 2014;20(1):22-29. DOI: 10.1177/1754045313515122.

13. McMillan E, Adan Sanchez A, Bhaduri A, et al. Sexual functioning and experiences in young people affected by mental health disorders. Psychiatry Res 2017;253:249-255. DOI: 10.1016/j.psychres.2017.04.009.

14. Singh JC, Tharyan P, Kekre NS, et al. Prevalence and risk factors for female sexual dysfunction in women attending a medical clinic in south India. J Postgrad Med 2009;55(2):113-120. DOI: 10.4103/00223859.52842.

15. Burri $A$, Hilpert $P$, Spector T. Longitudinal evaluation of sexual function in a cohort of pre- and postmenopausal women. J Sex Med 2015;12(6):1427-1435. DOI: 10.1111/jsm.12893.

16. Mishra VV, Nanda S, Gandhi K, et al. Female sexual dysfunction in patients with endometriosis: Indian scenario. J Hum Reprod Sci 2016;9(4):250-253. DOI: 10.4103/0974-1208.197687.

17. McCabe MP, Connaughton C. Sexual dysfunction and relationship stress: how does this association vary for men and women? Curr Opin Psychol 2016;13:81-84. DOI: 10.1016/j.copsyc.2016.05.007.

18. Garneau-Fournier J, McBain S, Torres T, et al. Sexual dysfunction problems in female college students: sexual victimization, substance use, and personality factors. J Sex Marital Ther 2015;43(1):24-39. DOI: 10.1080/0092623X.2015.1113595.

19. Tekin A, Meriç C, Sağbilge E, et al. The relationship between childhood sexual/physical abuse and sexual dysfunction in patients with social anxiety disorder. Nord J Psychiatry 2015;70(2):88-92. DOI: 10.3109/08039488.2015.1053097.

20. Sreelakshmy K, Velayudhan R, Kuriakose D, et al. Sexual dysfunction in females with depression: a cross-sectional study. Trends Psychiatry Psychother 2017;39(2):106-109. DOI: 10.1590/2237-6089-20160072.

21. Diehl A, Silva RL, Laranjeira R. Female sexual dysfunction in patients with substance-related disorders. Clinics (Sao Paulo) 2013;68(2): 205-212. DOI: 10.6061/clinics/2013(02)OA14.

22. Vanwesenbeeck I, Have MT, de Graaf R, et al. Associations between common mental disorders and sexual dissatisfaction in the general population. Br J Psychiatry 2014;205(2):151-157. DOI: 10.1192/bjp. bp.113.135335.

23. McCabe MP, Sharlip ID, Lewis $R$, et al. Incidence and prevalence of sexual dysfunction in women and men: a consensus statement from the fourth international consultation on sexual medicine 2015. J Sex Med 2016;13(2):144-152. DOI: 10.1016/j.jsxm.2015.12.034.

24. Graziottin A, Serafini A, Palacios S. Aetiology, diagnostic algorithms and prognosis of female sexual dysfunction. Maturitas 2009;63(2): 128-134. DOI: 10.1016/j.maturitas.2009.04.007. 
25. Brotto L, Atallah S, Johnson-Agbakwu C, et al. Psychological and interpersonal dimensions of sexual function and dysfunction. J Sex Med 2016;13(4):538-571. DOI: 10.1016/j.jsxm.2016.01.019.

26. Simiyon M, Chandra PS, Desai G. Sexual dysfunction among women with schizophrenia-a cross sectional study from India. Asian J Psychiatr 2016;24:93-98. DOI: 10.1016/j.ajp.2016.08.022.

27. Sørensen T, Giraldi A, Vinberg M. Sexual distress and quality of life among women with bipolar disorder. Int J Bipolar Disor 2017;5(1):29. DOI: 10.1186/s40345-017-0098-0.

28. Crisp C, Vaccaro C, Fellner A, et al. The influence of personality and coping on female sexual function: a population survey. J Sex Med 2014;12(1):109-115. DOI: 10.1111/jsm.12735.

29. Aksoy UM, Aksoy SG, Maner F, et al. Sexual dysfunction in obsessive compulsive disorder and panic disorder. Psychiatr Danub. 2012;24(4):381-385.

30. Dèttore D, Pucciarelli M, Santarnecchi E. Anxiety and female sexual functioning: an empirical study. J Sex Marital Ther 2013;39(3):216-240. DOI: 10.1080/0092623X.2011.606879.

31. Castellini G, Lelli L, Ricca V, et al. Sexuality in eating disorders patients: etiological factors, sexual dysfunction and identity issues. a systematic review. Horm Mol Biol Clin Investig 2016;25(2):71-90. DOI: 10.1515/hmbci-2015-0055.

32. Taegtmeyer AB, Krähenbühl S. Drug-induced sexual dysfunction. Ther Umsch. 2015;72(11-12):711-715. DOI: 10.1024/0040-5930/a000741.

33. Lorenz T, Rullo J, Faubion S. Antidepressant-induced female sexual dysfunction. Mayo Clin Proc 2016;91(9):1280-1286. DOI: 10.1016/ j.mayocp.2016.04.033.

34. de Boer MK, Castelein S, Wiersma D. The facts about sexual (Dys) function in schizophrenia: an overview of clinically relevant findings. Schizophr Bull 2015;41(3):674-686. DOI: 10.1093/schbul/sbv001.

35. Schmidt HM, Hagen M, Kriston L, et al. Management of sexual dysfunction due to antipsychotic drug therapy. Cochrane Database Syst Rev 2012;11:CD003546. DOI: 10.1002/14651858.CD003546.pub3.

36. Tufan AE, Ozten E, IsikS, et al. Discerning the effects of psychopathology and antidepressant treatment on sexual dysfunction. Int J Psychiatry Clin Pract 2013;17(3):223-226. DOI: 10.3109/13651501.2012.704382.
37. Allahdadi KJ, Tostes RC, Webb RC. Female sexual dysfunction: therapeutic options and experimental challenges. Cardiovasc Hematol Agents Med Chem 2009;7(4):260-269. DOI: 10.2174/187152509789541882.

38. Polland AR, Davis M, Zeymo A, et al. Association between comorbidities and female sexual dysfunction: findings from the third national survey of sexual attitudes and lifestyles (Natsal-3). Int Urogynecol J 2018;15(5):678-686. DOI: 10.1007/s00192-0183739-7.

39. Worsley R, Santoro N, Miller KK, et al. Hormones and female sexual dysfunction: beyond estrogens and androgens-findings from the fourth international consultation on sexual medicine. J Sex Med 2016;13(3):283-290. DOI: 10.1016/j.jsxm.2015.12.014.

40. Hatzichristou D, Kirana PS, Banner L, et al. Diagnosing sexual dysfunction in men and women: sexual history taking and the role of symptom scales and questionnaires. J Sex Med 2016;13(8):1166-1182. DOI: 10.1016/j.jsxm.2016.05.017.

41. Wåhlin-Jacobsen S, Kristensen E, Pedersen AT, et al. Androgens and psychosocial factors related to sexual dysfunctions in premenopausal women*: *2016 ISSM Female sexual dysfunction prize. J Sex Med 2017;14(3):366-379. DOI: 10.1016/j.jsxm.2016.12.237.

42. Nappi RE, Cucinella L. Advances in pharmacotherapy for treating female sexual dysfunction. Expert Opin Pharmacother 2015;16(6): 875-887. DOI: 10.1517/14656566.2015.1020791.

43. Taylor MJ, Rudkin L, Bullemor-Day P, et al. Strategies for managing sexual dysfunction induced by antidepressant medication. Cochrane Database Syst Rev 2013(5):CD003382. DOI: 10.1002/14651858. CD003382.pub3.

44. Thomas HN, Thurston RC. A biopsychosocial approach to women's sexual function and dysfunction at midlife: a narrative review. Maturitas 2016;87:49-60. DOI: 10.1016/j.maturitas.2016.02.009.

45. Waldinger MD. Psychiatric disorders and sexual dysfunction. Handb Clin Neurol 2015;130:469-489.

46. Bachmann G. Female sexuality and sexual dysfunction: are we stuck on the learning curve? J Sex Med 2006;3(4):639-645. DOI: 10.1111/j.1743-6109.2006.00265.x. 